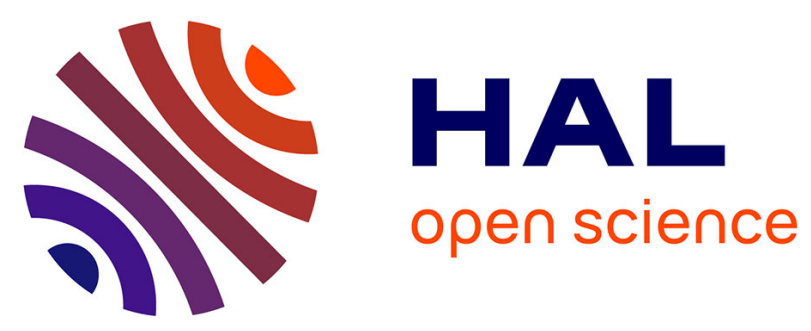

\title{
Sensitivity Analysis of a Left Ventricle Model in the Context of Intraventricular Dyssynchrony
}

Virginie Le Rolle, Elena Galli, David Danan, Karim El Houari, Arnaud Hubert, Erwan Donal, Alfredo I Hernández

\section{- To cite this version:}

Virginie Le Rolle, Elena Galli, David Danan, Karim El Houari, Arnaud Hubert, et al.. Sensitivity Analysis of a Left Ventricle Model in the Context of Intraventricular Dyssynchrony. Acta Biotheoretica, 2020, 68 (1), pp.45-59. 10.1007/s10441-019-09362-y . hal-02304379

HAL Id: hal-02304379

https://hal-univ-rennes1.archives-ouvertes.fr/hal-02304379

Submitted on 11 Mar 2021

HAL is a multi-disciplinary open access archive for the deposit and dissemination of scientific research documents, whether they are published or not. The documents may come from teaching and research institutions in France or abroad, or from public or private research centers.
L'archive ouverte pluridisciplinaire HAL, est destinée au dépôt et à la diffusion de documents scientifiques de niveau recherche, publiés ou non, émanant des établissements d'enseignement et de recherche français ou étrangers, des laboratoires publics ou privés. 
Noname manuscript No.

(will be inserted by the editor)

\title{
Sensitivity analysis of a left ventricle model in the context of intraventricular dyssynchrony
}

\author{
Virginie Le Rolle • Elena Galli • \\ David Danan - Karim El Houari • \\ Arnaud Hubert - Erwan Donal . \\ Alfredo I. Hernández
}

Received: date / Accepted: date

\begin{abstract}
The objective of the current study was to propose a sensitivity analysis of a 3D left ventricle model in order to assess the influence of parameters on myocardial mechanical dispersion. A finite element model (FEM) of LV electromechanical activity was proposed and a screening method was used to evaluate the sensitivity of model parameters on the standard deviation of time to peak strain (TPS-SD). Results highlight the importance of propagation parameters associated with septal and lateral segments activation. Simulated curves were compared to myocardial strains, obtained from echocardiography of one healthy subject and one patient diagnosed with intraventricular dyssynchrony and coronary artery disease. Results show a close match between simulation and clinical strains and illustrate the model ability to reproduce myocardial strains in the context of intraventricular dyssynchrony.
\end{abstract}

Keywords Cardiac Modeling · Cardiac mechanics · intraventricular dyssynchrony $\cdot$ clinical data

\footnotetext{
Virginie Le Rolle

Univ Rennes, CHU Rennes, Inserm, LTSI - UMR 1099, F-35000 Rennes, France

Tel.: +33223235903

Fax: +33223236917

E-mail: virginie.lerolle@univ-rennes1.fr

Elena Galli, David Danan, Karim El Houari, Arnaud Hubert, Erwan Donal, Alfredo I. Hernández

Univ Rennes, CHU Rennes, Inserm, LTSI - UMR 1099, F-35000 Rennes, France
} 


\section{Introduction}

In the normal left ventricle (LV), all segments contracts almost synchronously. In patients with LV conduction delay, for instance in the case of left bundle branch block (LBBB), LV dyssynchrony might occur, since some segments are stretched in systole whereas others contracts. Assessment of left ventricular mechanical dyssynchrony is typically based on the echocardiographic analysis of differences in timing of myocardial strains [1]. In particular, the standard deviation of time to peak strain (TPS-SD) has been proposed in the literature as an informative marker [2]. However, these methods neglect the electromechanical phenomena at the origin of cardiac contraction. In this context, a model-based approach can be used to improve the analysis of myocardial strain signals.

Mathematical modelling of the cardiac activity is an active field of research and the state-of-art is wide and very active. A variety of mathematical models of the ventricular function have been proposed in other studies [9]. The simplest models are based on a time-varying elastance $[10,11]$. Cardiac electrical activity could be represented by: $i$ ) biophysical continuous models at different levels of details [8], ii) phenomenological continuous models [36], or iii) simplified discrete models, often based on cellular automata [7]. Coupling of cell-level continuous models in order to represent a patch of myocardial tissue, or more complex geometries such as the whole LV, can be performed by using the monodomain or bidomain approaches $[6,5]$. Electrical activity models can be enriched to integrate the ventricular mechanical function, which is usually described as a function of the active [12,13] and passive [3,4] properties of myocardial tissue. In most cases, simulations of these models $[16,17,18]$ require significant computational resources, making these models difficult to analyze numerically and difficult to apply during clinical measurement protocols. As a consequence, there is still room for tissue-level LV models that may be numerically analyzed and applied into clinical practice.

The aim of the current study was to propose a sensitivity analysis of a $3 \mathrm{D}$ electro-mechanical model of cardiac activity in order to reproduce regional myocardial deformations in the case of intraventricular dyssynchrony. A tissue-level finite element model (FEM) of LV electro-mechanical activity was proposed in order to simulate myocardial strains corresponding to the standardized myocardial segmentation recommended by the American Heart Association. A screening sensitivity analysis method was applied to study the influence of electrical parameters on the TPS-SD, in order to determine the most influent parameters on strain signals and to define a reduced set of parameters, which could be identified in futur works. Finally, simulations were compared to strain morphologies observed in a healthy subject and a patient diagnosed with intraventricular dyssynchrony. The document is organized as follows. In Section 2, the clinical data and a description of the LV model, from the geometry to the multi-physics modeling and including the coupling procedures, are presented. In Sections 3 and 4, the results of applying the described methods are presented and discussed. 


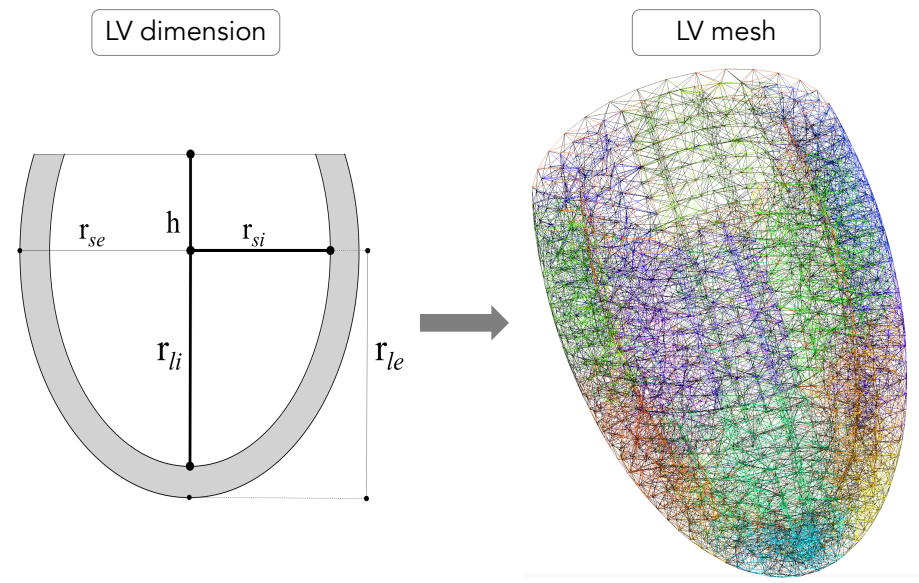

Fig. 1 creation of the mesh of the 3D LV model, which was divided in 16 segment according to the standardized segmentation of the AHA. Myocardial segments appears with different colors.

\section{Method}

\subsection{Model of the left ventricle}

\subsubsection{Geometry and segmentation}

In this work, the LV model geometry was approximated by a truncated ellipsoid (Figure 1), defined by an endocardial surface $\left(r_{s i}, r_{l i}\right)$, the epicardial surface $\left(r_{s e}, r_{l e}\right)$ and a base plane height $h$. These parameters were evaluated by fitting the truncated ellipsoid to the semi-automatic segmentation of the endocardial wall, extracted from apical 4 and 2 chamber-views. The uniformity of LV wall thickness was assumed to evaluate parameters $r_{s i}, r_{l i}$. A mesh was generated from this truncated ellipsoid by using gmsh (http://gmsh.info/). The left ventricle was divided into 16 segment, according to the standardized segmentation of the AHA [22]. The LV is firstly divided into basal (Bas), midcavity (Mid), and apical (Api) thirds. Each slice was then divided in order to consider the septum (Sept), the inferior (Inf), anterior (Ant) and lateral (Lat) walls.

\subsubsection{Electrical problem}

Propagation of the transmembrane potential $V_{m}$ was represented under the hypothesis of equal anisotropy ratio $[29,30,15]$ :

$$
\begin{aligned}
& \operatorname{div}\left(\sigma_{e q} \nabla V_{m}\right)=\chi\left(C_{m} \frac{\partial V_{m}}{\partial t}+I_{i o n}\left(V_{m}, w\right)\right) \quad \text { in } \Omega, \\
& \frac{\partial w}{\partial t}=g\left(V_{m}, w\right) \text { in } \Omega
\end{aligned}
$$


in which $\sigma_{e q}=\left(\sigma_{e}^{-1}+\sigma_{i}^{-1}\right)^{-1}, \sigma_{e}, \sigma_{i}, \sigma_{e q}$ represent, respectively, the extracellular, intracellular and equivalent electrical conductivities, $w$ is a state variable of the simplified ionic model, $C_{m}$ is the membrane surface capacitance, $\chi$ is the rate of cellular membrane surface per volume unit. The functions $I_{i o n}$ and $g$, representing simplified dynamics of the cellular action potential, are described as a modified version of the FitzHugh-Nagumo model (FHN) [34].

$$
\begin{aligned}
& I_{\text {ion }}\left(V_{m}, w\right)=k c_{1}\left(V_{m}-B\right)\left(a-\frac{V_{m}-B}{A}\right)\left(1-\frac{V_{m}-B}{A}\right)+k c_{2} w\left(V_{m}-B\right)(3) \\
& g\left(V_{m}, w\right)=k e\left(\frac{V_{m}-B}{A}-d w-b\right),
\end{aligned}
$$

where $a, b, c_{1}, c_{2}, d, e, k, A$ and $B$ are region specific parameters [31,15]. A point $N_{\text {exct }}$ of the domain $\Omega$ is electrically stimulated with a value $I_{\text {stim }}$ for a preset time $t_{e x c}(\boldsymbol{\nu}$ is the unit outward normal vector):

$$
-\left(\sigma_{e q} \nabla V_{m}\right) \cdot \boldsymbol{\nu}=\left\{\begin{aligned}
I_{s t i m}(t) & \text { if } t<t_{e x c} \\
0 & \text { else }
\end{aligned} \quad \text { on } N_{\text {exct }}\right.
$$

The electrical problem was solved with the finite element method (FEM) and a characteristic timing of the propagation $T_{s}$ was collected for each node of the mesh; it corresponds to the peak of transmembrane potential.

\subsubsection{Mechanical problem and electrical-mechanical coupling}

The Cauchy stress $\boldsymbol{\sigma}$ was described as the sum of a passive component $\boldsymbol{\sigma}_{p}$ and an active component $\sigma_{a}$ :

$$
\boldsymbol{\sigma}=\boldsymbol{\sigma}_{p}+\sigma_{a} \boldsymbol{I}
$$

In order to avoid the high computational resources required by hyperelastic mechanical constitutive laws [19], linear elasticity was assumed to describe the ventricle motion $[32,33]$.

$$
\left(\sigma_{p}\right)_{i j}=\lambda \varepsilon_{k k} \delta_{i j}+2 \mu \varepsilon_{i j}
$$

where we denote by $\lambda$ and $\mu$, the Lame coefficients, by $\delta_{i j}$ the Kronecker symbol and with

$$
\varepsilon_{i j}(\boldsymbol{v})=\frac{1}{2}\left(\frac{\partial v_{i}}{\partial x_{j}}+\frac{\partial v_{j}}{\partial x_{i}}\right)
$$

where $\boldsymbol{v}$ is the displacement vector. Active properties are initiated by the cardiac electrical activity. Electrical-mechanical coupling was described by phenomenological approaches [12,13], considering a time-varying analytical activation function that reach its minimum during diastole and its maximum 
during peak systole $[20,21]$. In this work, the active constraint was represented by a three-steps continuous Gaussian function:

$$
\sigma_{a}(t)=\left\{\begin{array}{l}
\sigma_{0} e^{-\psi_{1}\left(t-T_{s}\right)^{2}} \text { if } t \leq T_{s} \\
\sigma_{0} e^{-\psi_{2}\left(t-T_{s}\right)^{2}} \text { if } T_{s}<t \leq T_{c} \\
\sigma_{0} e^{\left(\psi_{3}-\psi_{2}\right)\left(T_{c}-T_{s}\right)^{2}} e^{-\psi_{3}\left(t-T_{s}\right)^{2}} \text { otherwise }
\end{array}\right.
$$

where $\sigma_{0}$ is the amplitude of the active constraint signal, $\psi_{1}$ is a growth parameter associated to the contraction phase, $\psi_{2}$ and $\psi_{3}$ are growth parameters associated to the relaxation phase. $T_{s}$ was extracted from the electrical problem. For each ventricular segment, it corresponds to the time associated with maximum electrical potential. $T_{c}$ was defined for each patient as the beginning of $\mathrm{P}$ wave deduced from the ECG. Within the cardiac wall, the equation of conservation of momentum was used while neglecting volumetric and inertial forces. To prevent rigid body motion, the displacement of the base to the apex direction is set to zero and the circumferential motion in the endocardial base of the LV is prevented (only radial motion is allowed).

\subsection{Extraction of myocardial strains}

The segmentation of the LV model allowed the definition of 16 couples of points, each of them belonging to a LV segment seg (Fig 2). The strain $S_{\text {seg }}(t)$ is defined by:

$$
S_{\text {seg }}(t)=\left(\frac{\left\|x_{\text {seg }, 1}(t)-x_{\text {seg }, 2}(t)\right\|}{\left\|X_{\text {seg }, 1}-X_{\text {seg }, 2}\right\|}-1\right) \times 100
$$

where $x_{s e g, 1}(t), x_{s e g, 2}(t)$ are the positions of 2 points of interest at a given time $t$ and $X_{\text {seg, } 1}, X_{\text {seg }, 2}$ are the positions of the same points in the reference configuration, which was defined as the beginning of the depolarisation of first node $N_{\text {exct }}$. This time could be considered as similar to the base of QRS complex that was considered as reference in experimental data. From simulated myocardial strains, the standard deviation of time to peak strain (TPS-SD) were calculated as proposed in [2].

The different physical problems and numerical methods were implemented in a code, which is based on a finite element library in $\mathrm{C}++$ under the GNU Public license: GEneric Tools for Finite Elements Methods (GETFEM++, https://savannah.nongnu.org/projects/getfem). The organization of the project is based on modules that must be run in a specific order so that the output produced by the module $n$ could be used as an input for the module $n+1$. The first two modules build the mesh and define LV segmentation that allows the calculation of strain signals. The following module solves the finite element electrical problem and computes the electrical propagation caracteristic timings used to generate the active constraint in the mechanical problem. Finally, a mechanical module solves the fully coupled problem, including the electromechanical coupling, and computes the strain signals, by using the strain points files. 

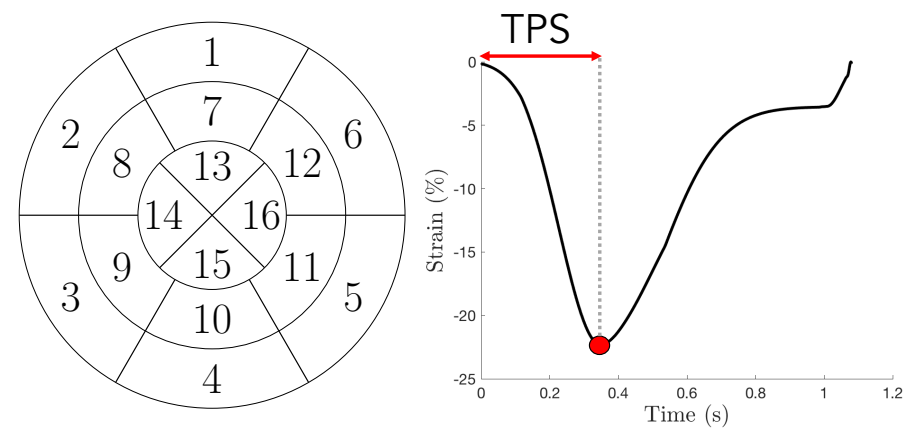

Fig. 2 LV segmentation (left panel): 1) basal anterior, 2) basal anteroseptal, 3) basal inferoseptal, 4) basal inferior, 5) basal inferolateral, 6) basal anterolateral, 7) mid anterior, 8) mid anteroseptal, 9) mid inferoseptal, 10) mid inferior, 11) mid inferolateral, 12) mid anterolateral, 13) apical anterior, 14) apical septal, 15) apical inferior, 16) apical lateral. Segmental strain and time to peak strain (TPS) (right panel)

\subsection{Sensitivity analysis method}

Using the Morris elementary effects method [14], the sensitivity of each parameter of the model $\left(X_{i}\right)$ on a given model output $Y$ can be estimated, by applying repeated simulations of $Y$ while changing one parameter at a time. Briefly, an elementary effect is defined as:

$$
\mathrm{EE}_{i}^{*}=\left|\frac{Y\left(X_{1}, \ldots, X_{i}, \ldots, X_{n}\right)-Y\left(X_{1}, \ldots, X_{i}+\Delta, \ldots, X_{n}\right)}{\Delta}\right|,
$$

where $\Delta$ is a predefined variation. For each parameter $X_{i}$, a number $r$ of elementary effects are calculated and the mean $\left(\mu^{*}\right)$ and standard deviation $(\sigma)$ of these elementary effects are then calculated. These values permit to determine which parameters are negligible (low $\mu^{*}$ and $\sigma$ ), linear (non-zero $\mu^{*}>\sigma$ ), and either non-linear or dependent on interactions with other parameters (nonzero $\left.\mu^{*} \leq \sigma\right)$. $Y$ was chosen equal to TPS-SD.

In this paper, we choose to analyse only the electrical parameters. In fact, heart failure with dyssynchronous LV contraction is most often related to a conduction block in the left bundle of the His. This blockade results in delayed electrical activation of the lateral LV and leads to abnormal contraction. As a consequence, it seems essential to first analyse the influence of cardiac electrical activity on myocardial strains. As we wish to limit computational cost, we have proceed to a first selection of parameters. Parameter $C_{m}$ is the membrane surface and could be considered to have a constant physical value. We have also excluded $c_{1}, c_{2}, d, e, k, A$ and $B$ because they appear to be less influent on electrical activity in a previous study [15]. Nevertheless, the method was applied on 3 parameters ( $\chi, a$ and $b$ ) for 16 segments, which represent a total of 48 parameters. Thus, the sensitivity analysis provide us a rank of importance reflecting both categories of parameters $(\chi, a$ and $b)$ and myocardial locations. Parameter ranges were selected from the nominal values $[15] \pm 30 \%$. As each 
cycle arising from the Morris elementary effects method involves completely independent computations, parallel computing using the MPI library was used in order to minimize the CPU time.

\subsection{Clinical data}

We studied data from 1 healthy subject and 1 patient, diagnosed with intraventricular dyssynchrony and coronary arteries disease (CAD). A standard TransThoracic Echocardiography (TTE) was performed using a Vivid E9 ultrasound system (General Electric Healthcare, Horten, Norway). Images were recorded on a remote station for off-line analysis by dedicated software (EchoPAC PC, version BT 13, General Electric Healthcare, Horten, Norway) and segmental strain signals were obtained from standard apical 4-chamber, 3 -chamber, and 2-chamber views. The study protocol was approved by our local ethics committee (authorization number: 2015-A00347-42).

\section{Results}

\subsection{Sensitivity analysis}

Figure 3 illustrates results of the sensitivity analysis. Parameters $a$ appear to be globally the most sensitive on TPS-SD, mainly due to their impact on intraventricular electrical activity propagation. Although $a$ and $b$ are difficult to relate to physical properties, since they arise from the FHN phenomenological model, the resulting sensitivity analysis highlight that their relative influence is highly region-specific. Sensitivity of $\chi$, which is a homogenization term, is associated with elevated standard-deviations $\left(\mu^{*}\right)$ in the Morris plane. Concerning $\chi, a$ or even $b$, basal septal segments are always more influent than other segments. Since these segments are the first to be depolarized in normal conditions, even the slightest modification of one of their parameters will modify the tissue depolarization front-wave, having a direct effect on the remaining segments. The sensitivity analysis shows that these parameters could be selected for subject-specific model identifications in future works.

A local sensitivity analysis on parameters $a$ is depicted in figure 4 . Inferior and lateral wall values of $a$ have been increased in order to study the influence on electrical propagation and strain morphologies. As a gets higher, strain curves from the lateral wall are delayed and a stretch morphology appears on their pre-systolic components. Indeed, in the case where $a$ lateral wall values are high (right part of the figure), the early systole is associated with a contraction of the septal segments (negative stain), whereas the opposite region, the lateral wall, is passively stretched (positive strain) due to the contraction of the septal wall. The delayed intraventricular electrical propagation results in an increased heterogeneity of strain curves and to a significant increase of TPS-SD. 


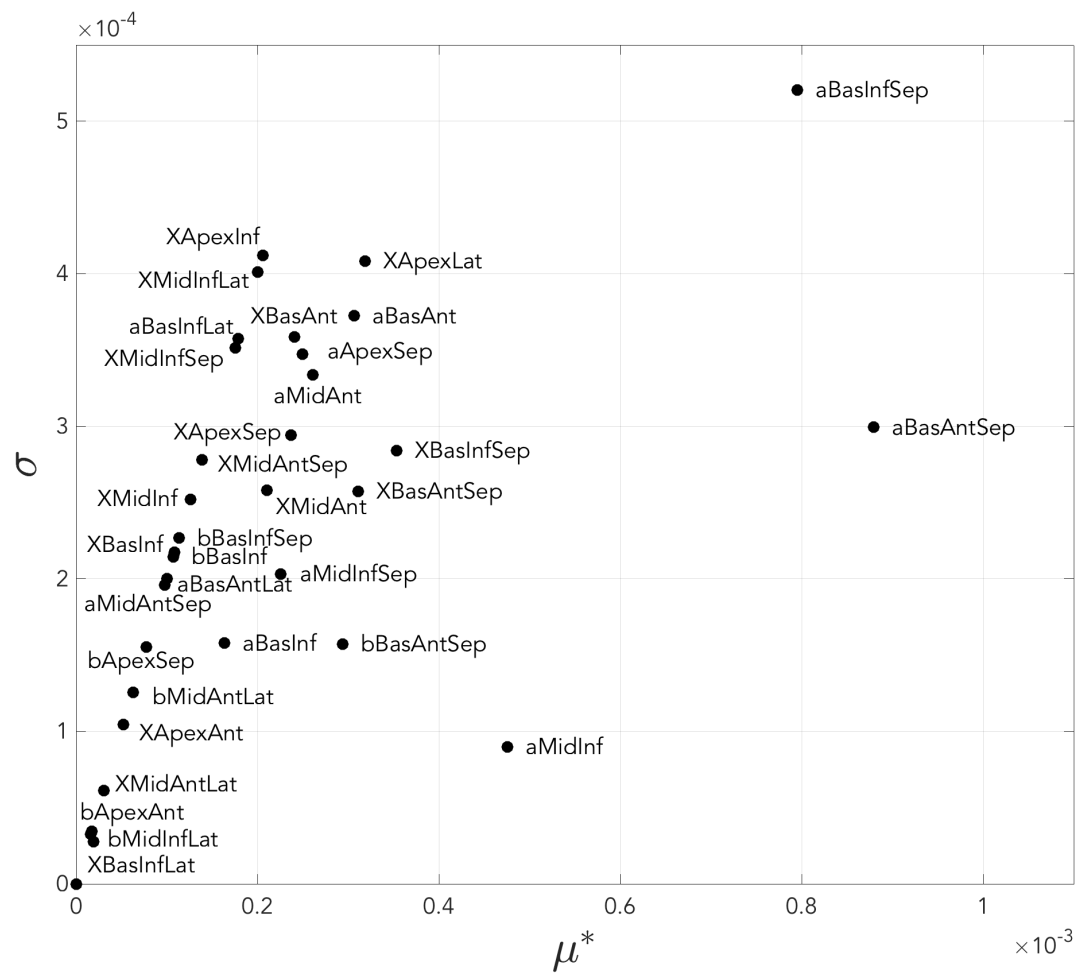

Fig. 3 Morris sensitivity analysis results. Mean $\left(\mu^{*}, \mathbf{s}\right)$ and standard deviation $(\sigma, \mathbf{s})$ of the elementary effects for the standard deviation of time to peak strain (TPS-SD). Only the most influential parameters are labeled.

\subsection{Analysis of myocardial strains in intraventricular dyssynchrony}

Figures 5 and 6 illustrate the simulated and experimental strains for a healthy subject and a patient diagnosed with intraventricular dyssynchrony and CAD. In this work, the parameter estimation was performed manually by varying the electrical $(a)$ and mechanical $\left(\sigma_{0}\right)$ parameters in order to obtain simulated strains similar to the observed clinical data. Parameter values were presented in figure 4 concerning $a$ and in the bottom panel of figures 5 and 6 concerning $\sigma_{0}$. Bull-eyes of figures 5 and 6 show the minimum strains, TPS and maximum stresses obtained from the simulated and experimental data. In both cases, although the morphologies are not completely reproduced, there is a close match between simulated and clinical minimal strain values. Correlations between simulated and experimental strain signals were on average $0.89( \pm 0.07)$ and $0.73( \pm 0.38)$ respectively for the 16 segments of the healthy subject and the patient diagnosed with intraventricular dyssynchrony and CAD. Root Mean Square Error (RMSE) were respectively equal to $3.22 \%( \pm 1.16)$ and 4.34 

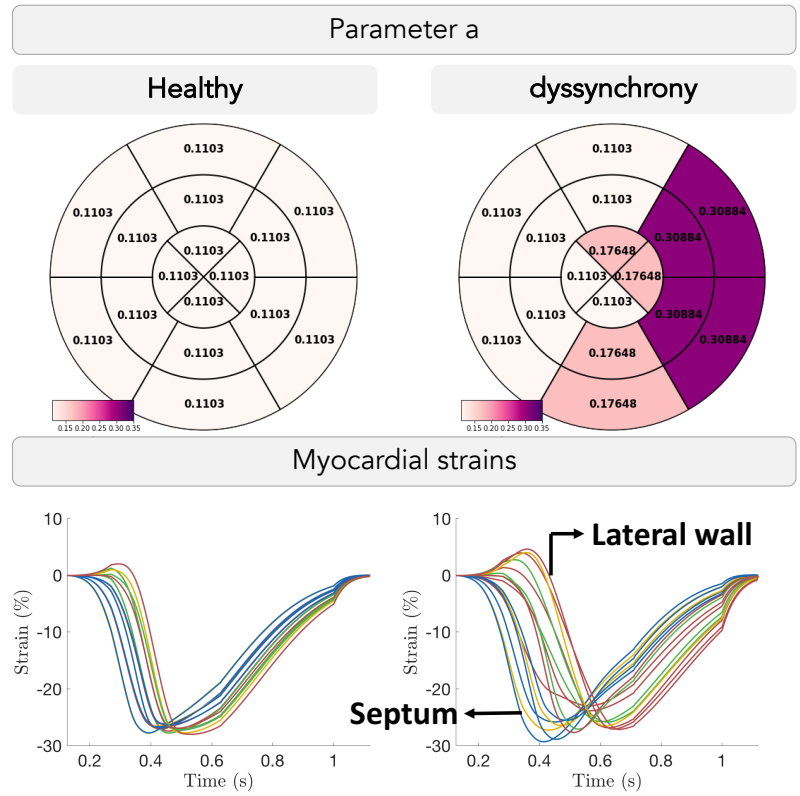

Fig. 4 Local sensitivity of parameters $a$ for each cardiac segment on corresponding myocardial strains. Two parameter sets, which reflect healthy and dyssynchrony cases, are presented. Top panel: Bull-Eyes depicting the $a$ parameter values of each segment. Bottom panel: 16 myocardial strain signals simulated during a whole cardiac cycle for the septal (red), anterior (yellow), inferior (green) and lateral (blue) walls are shown.

$\%( \pm 1.21)$. Concerning the healthy case, figure 5 shows the homogeneity of strain curves, which illustrates the synchronous contraction of left ventricle in normal subjects. Regarding the pathological case in figure 6, strain morphologies and minimum values are highly different depending on the myocardial segment. Septum values are more elevated compared with other segments and are associated with a decreased contractility.

\section{Discussion}

In this paper, a sensitivity analysis of a 3D LV model was proposed in order to evaluate the influence of electrical activity parameters on intraventricular dyssynchrony. The main contributions of this study concern: $i$ ) the application of a screening analysis method on parameters of a FEM model of cardiac electro-mechanical activity, ii) the comparison of simulated and experimental strain data for a healthy subject and a patient diagnosed with intraventricular dyssynchrony. Our results show the importance of propagation parameters associated with myocardial segments, and the feasibility of using a LV model to reproduce regional myocardial deformations in the case of dyssynchrony. 

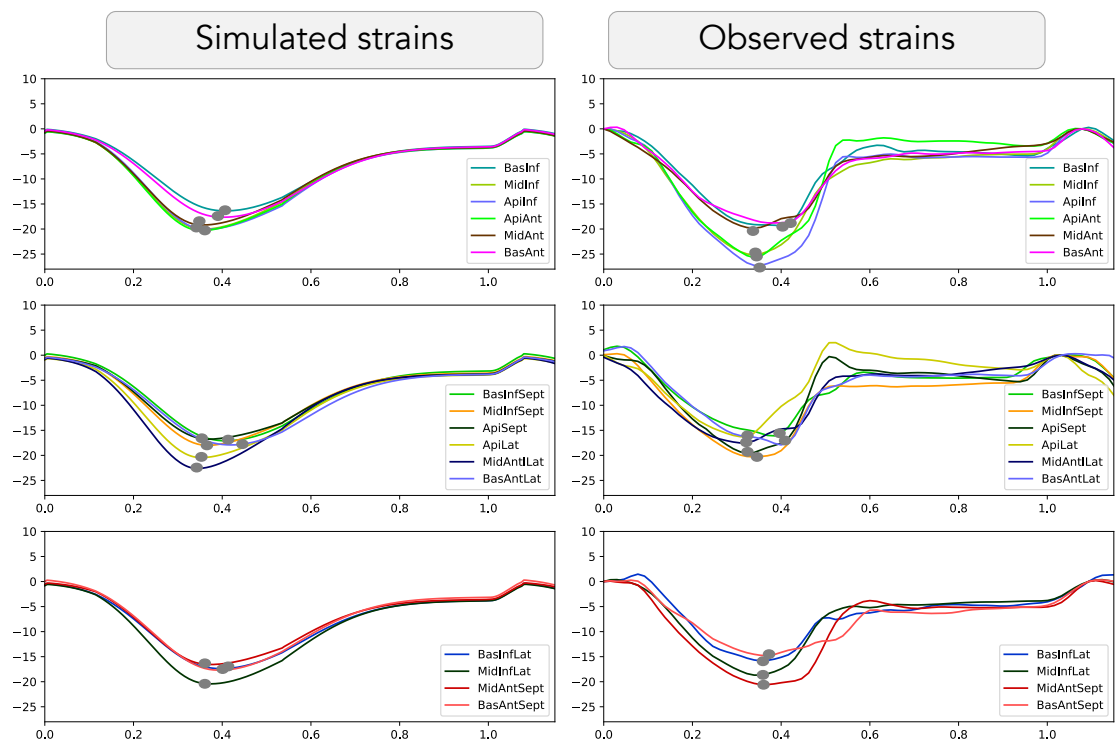

Simulated minimum strains (\%)

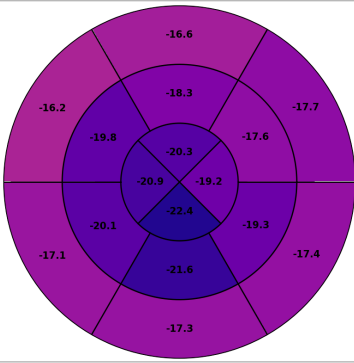

Simulated TPS (ms)

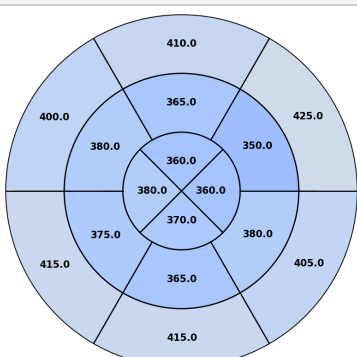

Simulated maximum stress $\left(\sigma_{0}, \mathrm{kPa}\right)$

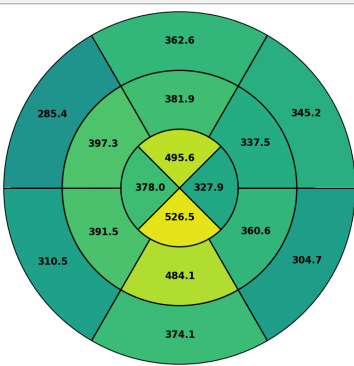

Data minimum strains (\%)

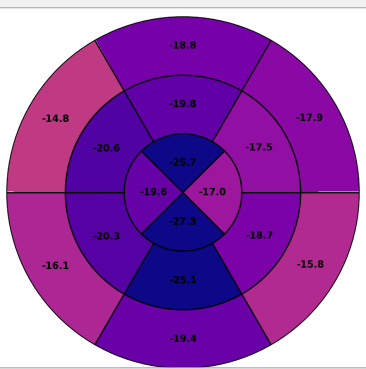

Data TPS (ms)

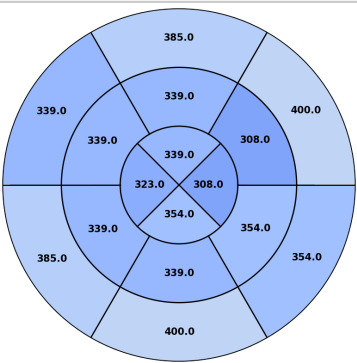

minimum strains $(\%)$
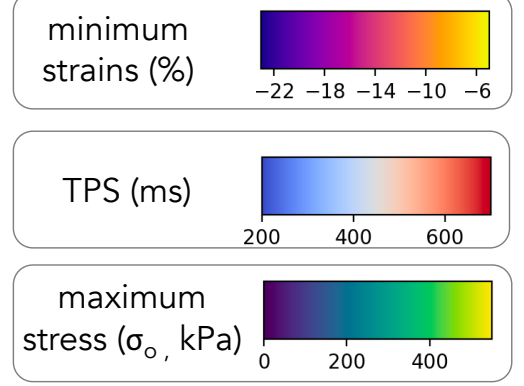

Fig. 5 Top panels: Comparison between simulated and experimental strain signals for a healthy case. 2C, 4C, APLAX stand respectively for 2 cavities, 4 cavities and apical longaxis views in echocardiography. Grey markers indicates minimum values of strain. Bottom panels: Bull-eye plots of minimum strains, TPS and maximum stresses. 

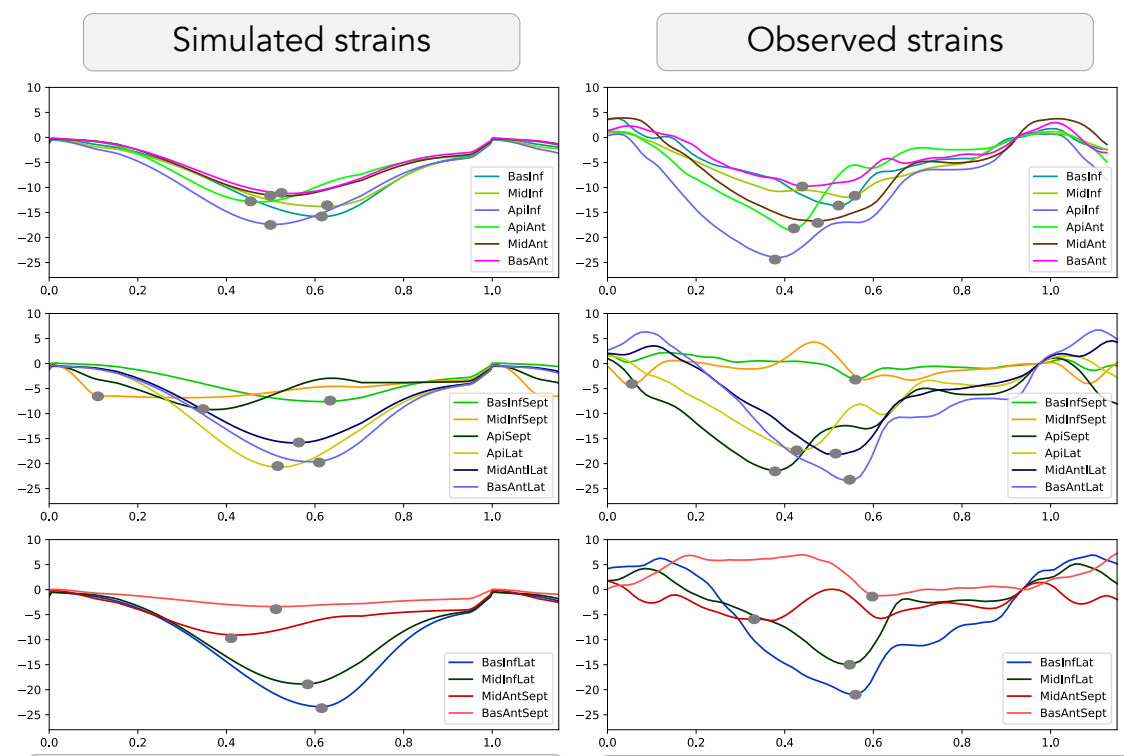

Simulated minimum strains (\%)

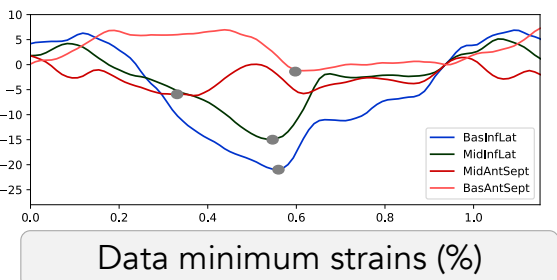

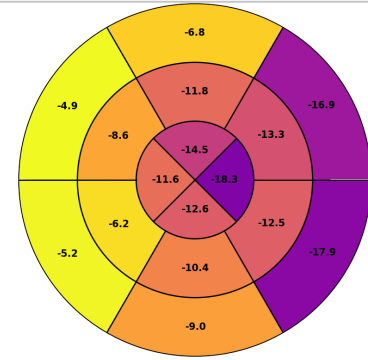

Simulated TPS (ms)

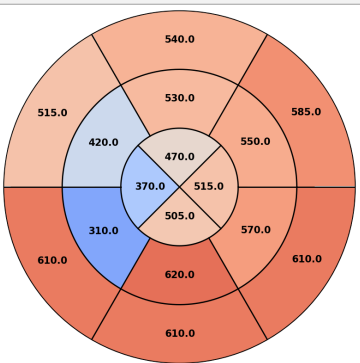

Simulated maximum stress $\left(\sigma_{\circ}, \mathrm{kPa}\right)$

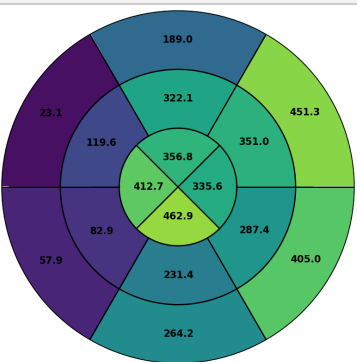

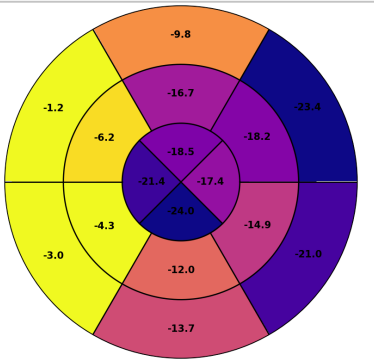

Data TPS (ms)

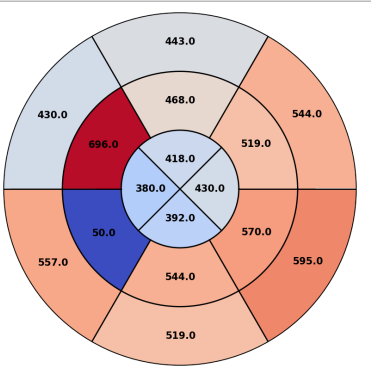

minimum strains $(\%)$
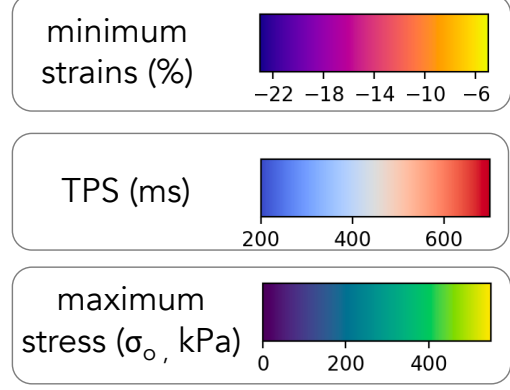

Fig. 6 Top panels: Comparison between simulated and experimental strain signals for a patient diagnosed with intra-ventricular dyssynchrony and ischemia. 2C, 4C, APLAX stand respectively for 2 cavities, 4 cavities and apical long-axis views in echocardiography. Grey markers indicates minimum values of strain. Bottom panels: Bull-eye plots of minimum strains, TPS and maximum stresses. 
Intraventricular dyssynchrony occurs when LV activation sequence is disrupted, resulting in discoordinated contraction of the LV segments. Recent studies have suggested the essential role of assessing systolic asynchrony by echocardiography in order to predict improvement of systolic function [23]. Myocardial mechanical dispersion, which could be quantified using the TPS-SD marker, has shown to be promising to assess systolic asynchrony and quantify risk in patients with impaired LV function [1]. Our sensitivity analysis results show that mechanical dispersion is highly influenced by parameters $a$ and $\chi$ of septal and lateral segments. Most basal segments, including anterior and inferior walls, are also particularly involved in the disparity of the septo-lateral wall. As a consequence, modifications of the electrical substrate of septal and lateral segments could induce heterogeneous activation of the LV. The electrical part of the 3D LV model is based on the adapted monodomain formalism together with the FitzHugh-Nagumo representation. Although it is based on simplified assumptions on the transmembrane potential fields, this approach has been widely used in previous works $[34,36,35,31,34,37]$ because it allows a reduction of: $i$ ) the computational costs and ii) the number of parameters that need to be tuned. To our knowledge, our work proposes the first sensitivity analysis of monodomain model, coupled to FitzHugh-Nagumo equations on myocardial strain signals. Although all parameters do not have a direct physical interpretation, our results is an important step toward the proposition of personalized models. In fact, it is possible to propose a reduced set of parameters that need to be identify to propose patient specific models.

Comparisons between simulated and experimental signals, shown in figure 5 and 6 , illustrate the model ability to reproduce characteristic strains patterns in healthy and cardiac dyssynchrony cases. The patient, with cardiac dyssynchrony, was simulated by modifying both myocardial contractility of septal wall and electrical parameters, especially in lateral segments. By comparing the healthy and dyssynchrony curves, mechanical dispersion becomes evident and is directly related to the electrical parameter values of each segment. In the desynchronized case, lateral segments are delayed, whereas septal wall seems to be activated prematurely. Interestingly, in the patient with LV dyssynchrony, a septal rebound can be observed, whereas the lateral wall has a typical systolic pre-stretch. The early onset of septal activation relative to the lateral wall, and its morphology is largely influenced by the septal and lateral wall contractility, which explains the low absolute value of strain peaks in the septal region $[24,25,26]$. The lateral wall stretch, on the contrary, is due to the early septal activation [27]. This systolic pre-stretch occurs before aortic valve opening and further delays the shortening of the lateral segments [28].

The proposed model and analyses in this work present a number of limitations that should be mentioned. The first limitation is the lack of a model of the right ventricle, which could highly influence myocardial strain morphology in the case of interventricular dyssynchrony. Our future work will focus on the integration of left and right ventricular interactions. A second limitation is related to the manual adjustment of model parameters, which should be replaced by an identification algorithm, as performed in our previous publications [20]. 
Moreover, the analysis was here limited to the parameters of the electrical activity. Parameter analyses of electro-mechanical coupling and the mechanical functions would provide valuable information to further improve the strain curve morphologies. Finally, concerning the clinical evaluation of the approach, only a limited number of subjects have been analyzed. Although these results are encouraging and show the feasibility of the proposed model to reproduce segmental strains, a larger clinical evaluation is necessary in order to validate the interpretations obtained from this model-based approach.

\section{Conclusion}

A sensitivity analysis method was applied to a LV model that integrates a 3D description of cardiac electro-mechanical activity. This work contributes to the identification of important parameters that should be selected for future parameter estimations or further improvements of the underlying mathematical descriptions. A preliminary patient-specific parameter estimation showed that the model can represent a significant intraventricular dyssynchrony, while staying stable and able to simulate myocardial strains that are qualitatively similar to those obtained from echocardiography. Further work will focus on the representation of right ventricular function, an analysis of the electro-mechanical parameters and the application of an automated estimation procedure in order to identify model parameters specifically to each patient.

\section{Funding}

French National Research Agency (ANR) (ANR-16-CE19-0008-01) (project MAESTRo).

\section{References}

1. Yu CM, Zhang Q, Fung JW, Chan HC, Chan YS, Yip GW, Kong SL, Lin H, Zhang Y, Sanderson JE. A novel tool to assess systolic asynchrony and identify responders of cardiac resynchronization therapy by tissue synchronization imaging. J Am Coll Cardiol. 2005 Mar 1;45(5):677-84.

2. Bertola B, Rondano E, Sulis M, Sarasso G, Piccinino C, Marti G, Devecchi P, Magnani A, Francalacci G, Marino PN. Cardiac dyssynchrony quantitated by time-to-peak or temporal uniformity of strain at longitudinal, circumferential, and radial level: implications for resynchronization therapy. J Am Soc Echocardiogr. 2009 Jun;22(6):665-71. doi: 10.1016/j.echo.2009.03.010. Epub 2009 May 7.

3. J. D. Humphrey, R. K. Strumpf, and F. C. Yin. Determination of a constitutive relation for passive myocardium : Ii. parameter estimation. Journal of Biomechanical Engineering, 112(3) :340?6, 1990.

4. P. J. Hunter. Myocardial constitutive laws for continuum mechanics models of the heart. Adv Exp Med Biol, 382 :303?18, 1995.

5. P. L. Colli Franzone, P. and T. B. Monodomain simulations of excitation and recovery in cardiac blocks with intramural heterogeneity. Funcional Imaging and Modeling of the Heart (FIMH), page 267?277, 2005. 
6. C. S. Henriquez and R. Plonsey. Simulation of propagation along a cylindrical bundle of cardiac tissue?i : Mathematical formulation. IEEE Transactions on Biomedical Engineering, 37(9) :850?60, 1990 .

7. Hernandez A, Carrault G, Mora F. Model-based interpretation of cardiac beats by evolutionary algorithms: signal and model interaction. Artificial Intelligence in Medicine. 2002;26(3):211?235

8. C. H. Luo and Y. Rudy. A dynamic model of the cardiac ventricular action potential ii. afterdepolarizations, triggered activity, and potentiation. Circulation Research, 74(6) :1097?113, 1994

9. I. V. Kazbanov, R. H. Clayton, M. P. Nash, C. P. Bradley, D. J. Paterson, M. P. Hayward, P. Taggart, and A. V. Panfilov. Effect of global cardiac ischemia on human ventricular fibrillation : insights from a multi-scale mechanistic model of the human heart. PLoS Comput Biol, 10(11) :e1003891, 2014.

10. M. Guarini, J. Urzua, A. Cipriano, and W. Gonzalez. Estimation of cardiac function from computer analysis of the arterial pressure waveform. IEEE Transactions on Biomedical Engineering, 45(12) :1420?8, 1998.

11. J. L. Palladino and A. Noordergraaf. A paradigm for quantifying ventricular contraction. Cellular and Molecular Biology Letters, 7(2) :331?5, 2002.

12. P. H. Bovendeerd, P. Borsje, T. Arts, and F. N. van De Vosse. Dependence of intramyocardial pressure and coronary flow on ventricular loading and contractility : a model study. Annals Biomedical Engineering, 34(12) :1833?45, 2006.

13. R. C. Kerckhoffs, P. H. Bovendeerd, J. C. Kotte, F. W. Prinzen, K. Smits, and T. Arts. Homogeneity of cardiac contraction despite physiological asynchrony of depolarization : a model study. annals of biomedical engineering, 31(5):536?47, 2003.

14. M. Morris, ?Factorial sampling plans for preliminary computational experiments,? Technometrics, vol. 33, no. 2, pp. 161?174, May 1991.

15. El Houari, K., Kachenoura A., Albera, L., Bensaid, S., Karfoul, A., Boichon-Grivot, C., Rochette, M., AND Hernandez, A. A fast model for solving the ECG forward problem based on an evolutionary algorithm. In Computational Advances in Multi-Sensor Adaptive Processing (CAMSAP), 2017 IEEE 7th International Workshop on (2017), IEEE, pp. 1?5.

16. Talbot, H., Marchesseau, S., Duriez, C., Sermesant, M., Cotin, S., and Delingette, H., Towards an interac- tive electromechanical model of the heart, Interface Focus 3(2), 20120091 (2013).

17. de Vecchi, A., Nordsletten, D. A., Razavi, R., Greil, G., and Smith, N. P., Patient specific fluid-structure ventricular modelling for integrated cardiac care, Med Biol Eng Comput 51(11), 1261-70 (2013).

18. McCormick, M., Nordsletten, D., Kay, D., and Smith, N., Simulating left ventricular fluid solid mechanics through the cardiac cycle under lvad support, 244, 80-96 (2013).

19. Danan D, Le Rolle V., Hubert A, Galli E, Bernard A, Donal E, and Hernandez A.I.(2017) Validation of a Finite Element Method framework for cardiac mechanics applications. SIPAIM 2017. 13th International Symposium on Medical Information Processing and Analysis, San Andres Island, Colombia, October 5 - 7, 2017

20. Le Rolle V., Hernandez A.I., Richard P.-Y., Donal E. and Carrault G. (2008) ModelBased Analysis of Myocardial Strain Data acquired by Tissue Doppler Imaging, In Artificial Intelligence in Medicine, 44(3) 201-19

21. Le Rolle V., Hernandez A.I., Richard P.-Y., P. Pibarot, L.-G. Durand and Carrault G. (2009) A tissue- level electromechanical model of the left ventricle : Application to the analysis of intraventricular pressure, Acta Biotheor, $57: 457-478$

22. Cerqueira MD, Weissman NJ, Dilsizian V, Jacobs AK, Kaul S, Laskey WK, Pennell DJ, Rumberger JA, Ryan T, Verani MS; American Heart Association Writing Group on Myocardial Segmentation and Registration for Cardiac Imaging. Standardized myocardial segmentation and nomenclature for tomographic imaging of the heart. A statement for healthcare professionals from the Cardiac Imaging Committee of the Council on Clinical Cardiology of the American Heart Association. Circulation. 2002 Jan 29;105(4):539-42.

23. Pitzalis MV, Iacoviello M, Romito R, et al. Cardiac resynchronization therapy tailored by echocardiographic evaluation of ventricular asynchrony. J Am Coll Cardiol 2002;40:1615?22. 
24. Walmsley J, Huntjens PR, Prinzen FW, Delhaas T, Lumens J. Septal flash and septal rebound stretch have different underlying mechanisms. Am J Physiol Heart Circ Physiol. 2016 Feb 1;310(3):H394-403. doi: 10.1152/ajpheart.00639.2015. Epub 2015 Dec 31.

25. Khan FZ, Virdee MS, Palmer CR, Pugh PJ, O'Halloran D, Elsik M, Read PA, Begley D, Fynn SP, Dutka DP. Targeted left ventricular lead placement to guide cardiac resynchronization therapy: the TARGET study: a randomized, controlled trial. J Am Coll Cardiol. 2012; 59(17):1509-18. doi: 10.1016/j.jacc.2011.12.030.

26. van Everdingen WM, Walmsley J, Cramer MJ, van Hagen I, De Boeck BWL, Meine M, Delhaas T, Doevendans PA, Prinzen FW, Lumens J, Leenders GE. Echocardiographic Prediction of Cardiac Resynchronization Therapy Response Requires Analysis of Both Mechanical Dyssynchrony and Right Ventricular Function: A Combined Analysis of Patient Data and Computer Simulations. J Am Soc Echocardiogr. 2017; 30(10):1012-1020.e2. doi: 10.1016/j.echo.2017.06.004.

27. Lumens J, Tayal B, Walmsley J, Delgado-Montero A, Huntjens PR, Schwartzman D, Althouse AD, Delhaas T, Prinzen FW, Gorcsan J 3rd. Differentiating Electromechanical From Non-Electrical Substrates of Mechanical Discoordination to Identify Responders to Cardiac Resynchronization Therapy. Circ Cardiovasc Imaging. 2015; 8(9):e003744. doi: 10.1161/CIRCIMAGING.115.003744.

28. Sweeney MO, Prinzen FW. Ventricular pump function and pacing: physiological and clinical integration. Circ Arrhythm Electrophysiol. 2008;1(2):127-39. doi: 10.1161/CIRCEP.108.777904.

29. L. Gerardo-Giorda, M. Perego, and A. Veneziani. Optimized schwarz coupling of bidomain and monodomain models in electrocardiology. M2AN, 2010.

30. J.P. Keener, Direct activation and defibrillation of cardiac tissue. J. Theor. Biol. 178 (1996) $313 ? 324$

31. Sovilj S, Magjarevic, Lovell NH, Dokos S. Comput Math Methods Med 2013;2013:134208. doi: 10.1155/2013/134208. Epub 2013 Apr 22. A simplified 3D model of whole heart electrical activity and 12-lead ECG generation.

32. Weise L.D., Panfilov A.V. A Discrete Electromechanical Model for Human Cardiac Tissue: Effects of Stretch-Activated Currents and Stretch Conditions on Restitution Properties and Spiral Wave Dynamics https://doi.org/10.1371/journal.pone.0059317

33. Sermesant, M., Delingette, H., and Ayache, N., "An electromechanical model of the heart for image analysis and simulation," ieee transactions on medical imaging 25(5), 612-25 (2006)

34. S. Dokos, S. L. Cloherty, and N. H. Lovell, Computational model of atrial electrical activation and propagation, in Engineering in Medicine and Biology Society, 2007. EMBS 2007. 29th Annual International Conference of the IEEE. IEEE, 2007, pp. 908911.

35. Karl Kunisch, Diego A. Souza, On the One-Dimensional Nonlinear Monodomain Equations with Moving Controls. Journal de Mathématiques Pures et Appliquées, 117 (9), pp 94-122 (2018)

36. FitzHugh R., Impulses and physiological states in theoretical models of nerve membrane, Biophysical J. 1:445-466 (1961)

37. J. M. Rogers and A. D. McCulloch, A collocation-Galerkin nite element model of cardiac action potential propagation, IEEE Transactions on Biomedical Engineering, 41 (1994), pp. 743-757. 